\title{
Slowing light with Fabry-Perot resonator arrays
}

\author{
Joyce K. S. Poon,* Philip Chak, John M. Choi, and Amnon Yariv \\ Department of Electrical Engineering and Department of Applied Physics, California Institute of Technology, \\ MS 128-95, Pasadena, California 91125, USA \\ *Corresponding author: joyce.poon@utoronto.ca
}

Received May 2, 2007; revised August 3, 2007; accepted September 4, 2007; posted September 10, 2007 (Doc. ID 82648); published October 1, 2007

\begin{abstract}
We analyze the transmission of light through coupled-resonator optical waveguides in the form of evanescently coupled Fabry-Perot resonator arrays. We develop a transfer matrix method to calculate the amplitude and phase responses of the arrays. We also discuss the inclusion of optical gain in the system to compensate for losses in these structures. Owing to the compact length along the propagation direction in evanescently coupled arrays, large slowing factors of the order of $10^{2}-10^{3}$ can be achieved even with a weak index contrast of $\sim 0.1 \%$. The large slowing factor, coupled with weak index contrast, makes this structure a promising candidate for artificial slow light system. (C) 2007 Optical Society of America

OCIS codes: $230.5750,130.2790$.
\end{abstract}

\section{INTRODUCTION}

A coupled-resonator optical waveguide (CROW) is a periodic array of resonators in which light propagates due to the weak coupling between nearest neighbors [1]. In artificially structured materials, the interresonator coupling can be made small by tuning the intercavity coupling spacing. As a result, CROWs have the potential to significantly slow down the propagation of light, which may find applications such as optical delay lines, interferometers, optical buffers, and nonlinear optics [2-5].

To achieve significant slowing in CROWs, and indeed in any medium, the optical delay should be achieved over as short a device length as possible in the direction of propagation. In many realizations of CROWs, such as coupled Bragg grating defects [6], photonic crystal defect cavities [7], or ring resonators [5,8,9], maximizing the slowing factor necessitates using a high refractive index contrast material system to keep the resonators compact and the interresonator coupling strength weak.

However, a high refractive index contrast poses some practical challenges. First, a high-index contrast significantly increases scattering loss due to sidewall roughness. Second, more complex fabrication procedures may be required for the devices. For example, small (submicrometer) feature sizes and a large etch depth (approximately micrometers) or even suspended membranes as in photonic crystal cavities may be needed [10]. Third, the high-index contrast can lead to a greater modal size and effective index mismatch between an optical fiber and the mode of the CROW, further increasing the insertion losses of the system.

Here we propose to use an array of evanescently coupled Fabry-Perot resonators as a low-index contrast slow light structure. Despite the low-index contrast, a high slowing factor is obtained by decoupling the length of the device in the propagation direction from the size of the resonators. The CROW consists of an array of linear waveguides terminated by reflectors in the direction perpendicular to the periodicity, so the waveguides become
Fabry-Perot resonators. Certain implementations of the CROW are depicted in Figs. 1(a) and 1(b). Although similar structures have been studied for discrete solitons, we will be analyzing slow light propagation in these structures [11]. A large slowing factor is possible because along $z$, the direction of propagation, the period of the device, $\Lambda$, can be short, say $\sim 5 \mu \mathrm{m}$ for evanescently coupled singlemode waveguides $(2-3 \mu \mathrm{m}$ wide waveguides spaced $\sim 1 \mu \mathrm{m}$ apart). The periodicity of the structure is similar to what is achievable in high-index contrast photonic crystal, ring, or disk resonators. In the $y$ direction, propagating optical waves are resonant with the cavities. Moreover, optical gain and electronic control can be readily incorporated into the coupled waveguide array, by leveraging diode laser array techniques $[12,13]$.

To excite the structure, an optical signal can be input to the resonator array in a side-coupled [Fig. 1(c)] or endcoupled [Fig. 1(d)] configuration. The output can be outcoupled in a similar manner out of the last element of the array. In the side-coupled scheme [Fig. 1(c)], the first and last element, or equivalently the input and output ports, of the structure do not possess reflectors and hence behave only as waveguides. In the end-coupled scheme [Fig. $1(\mathrm{~d})$ ], every element of the structure is a resonator. The differences in the input and output coupling mechanisms and configurations lead to a qualitative change of the transmission properties.

This paper presents a transfer matrix analysis of CROWs in the form of low-index contrast coupled FabryPerot resonator arrays. These CROWs have the potential to slow light hundreds of times compared to the free-space velocity. A high slowing factor compared to other systems is obtained because of its geometric configuration. We will first show how the conventional coupled mode approach commonly used to analyze waveguide arrays [13] can be extended to the treatment of coupled Fabry-Perot resonators. Using the technique outlined here, the dispersion properties of coupled Fabry-Perot resonators can be characterized by a few parameters. We will show how the 


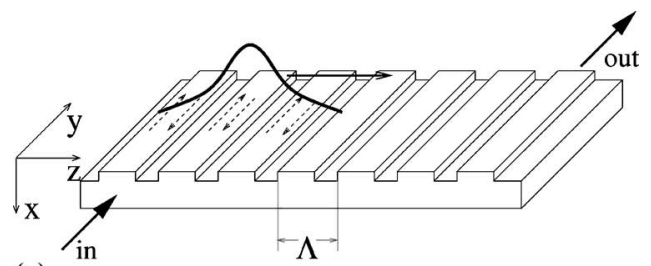

(a)

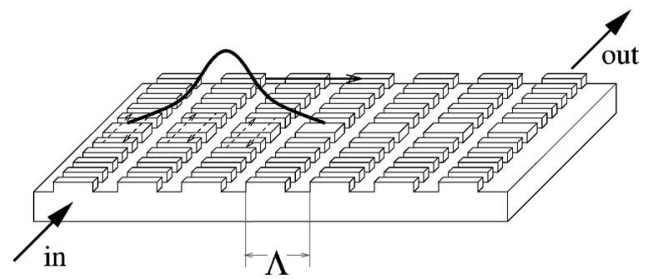

(b)

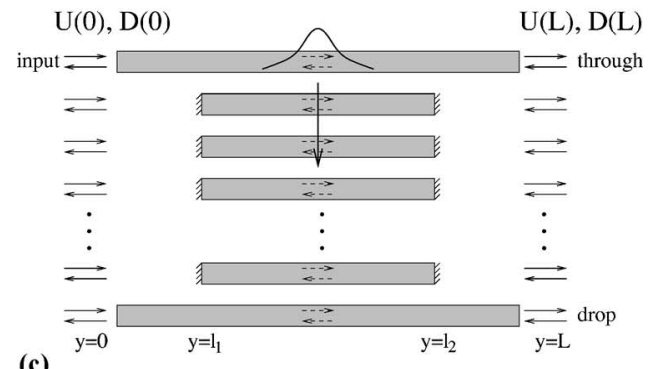

(c)

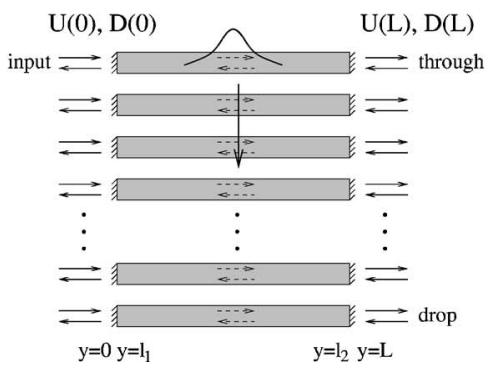

Fig. 1. Schematic of (a) waveguide laser and (b) DFB laser arrays in a planar geometry as implementations of CROWs. The input-output can be (c) side coupled or (d) end coupled into-out of the array. $\Lambda$ is the period of the CROW. The slanted lines represent reflectors that define each resonator. The arrows indicate the field propagation direction inside each resonator or waveguide. An optical pulse in the structure propagates in the direction of periodicity of the resonators.

transfer matrix formalism can be used to easily study arrays with side-coupled or end-coupled input and output ports. Finally, we will discuss the dispersion and transmission in the presence of optical gain.

\section{COUPLED-MODE THEORY}

Weakly coupled waveguide arrays are commonly analyzed using coupled-mode theory [13]. In this section, we will briefly review the approach and show how the dispersion relation of a tight-binding form [1] can be recovered from the analysis.

Using the coordinate system in Fig. 1, for an array of $N$ coupled, identical waveguides, we write the dielectric constant of the structure as

$$
\epsilon(\mathbf{r})=\bar{\epsilon}(\mathbf{r})+\sum_{n=1}^{N} \Delta \epsilon\left(\mathbf{r}_{\perp}-n \Lambda \hat{z}\right)
$$

where $\overline{\boldsymbol{\epsilon}}(\mathbf{r})$ is the dielectric constant in the absence of any waveguides, $\mathbf{r}_{\perp}$ represents the transverse coordinates $(x, z), \Delta \epsilon\left(\mathbf{r}_{\perp}\right)$ defines each waveguide, and $\Lambda$ is the period in the $z$ direction. In coupled-mode theory, we write the total field as a superposition of the modes of the constituent waveguides,

$$
\mathcal{E}(\mathbf{r})=\sum_{n=1}^{N} c_{n}(y) \mathcal{E}_{n}(x, z) \exp \left(-i \beta_{0} y\right),
$$

where $\mathcal{E}(\mathbf{r})$ is electric field in the array, $c_{n}(y)$ are coefficients of expansion, and $\mathcal{E}_{n}(x, z)$ and $\beta_{0}=\omega n_{\mathrm{eff}}(\omega) / c$ are the mode profile and propagation constant of the $n$th waveguide in the uncoupled case, respectively. $\omega$ is the optical frequency and $n_{\text {eff }}(\omega)$ is the effective index of the mode.

Expressing the $y$-dependent part of the total field as a column vector, we write

$$
\mathbf{E}(y) \equiv\left[\begin{array}{c}
c_{1}(y) e^{-i \beta_{0} y} \\
c_{2}(y) e^{-i \beta_{0} y} \\
\vdots \\
\vdots \\
\vdots \\
c_{N}(y) e^{-i \beta_{0} y}
\end{array}\right] \equiv\left[\begin{array}{c}
E_{1}(y) \\
E_{2}(y) \\
\vdots \\
\vdots \\
\vdots \\
E_{N}(y)
\end{array}\right] .
$$

Neglecting interaction between nonneighboring waveguides, the coupled-mode equations can thus be written in matrix form as

$$
\frac{\mathrm{d} \mathbf{E}}{\mathrm{d} y}=\mathbf{C E},
$$

where

$$
\mathbf{C}=-i\left[\begin{array}{ccccccc}
\beta_{0}+M_{l} & \kappa_{l} & 0 & 0 & \cdots & 0 & 0 \\
\kappa_{l} & \beta_{0}+M_{l} & \kappa_{l} & 0 & \cdots & 0 & 0 \\
\vdots & \vdots & \vdots & \vdots & \vdots & \vdots & \vdots \\
\vdots & \vdots & \vdots & \vdots & \vdots & \vdots & \vdots \\
\vdots & \vdots & \vdots & \vdots & \vdots & \kappa_{l} & \beta_{0}+M_{l}
\end{array}\right]
$$

Here $\kappa_{l}$ is the per length nearest-neighbor coupling coefficient and $M_{l}$ is the per length self-coupling coefficient given by

$$
\kappa_{l}=\frac{\omega \epsilon_{0}}{4} \int_{-\infty}^{\infty} \mathcal{E}_{n}^{*}\left(\mathbf{r}_{\perp}\right)\left[\epsilon\left(\mathbf{r}_{\perp}\right)-\Delta \epsilon(\mathbf{r}-n \Lambda)\right] \mathcal{E}_{n+1}\left(\mathbf{r}_{\perp}\right) \mathrm{d} \mathbf{r}_{\perp},
$$

$$
M_{l}=\frac{\omega \epsilon_{0}}{4} \int_{-\infty}^{\infty} \mathcal{E}_{n}^{*}\left(\mathbf{r}_{\perp}\right)\left[\epsilon\left(\mathbf{r}_{\perp}\right)-\Delta \epsilon(\mathbf{r}-n \Lambda)\right] \mathcal{E}_{n}\left(\mathbf{r}_{\perp}\right) \mathrm{d} \mathbf{r}_{\perp},
$$

where we have used the normalization

$$
\frac{\beta_{0}}{2 \omega \mu} \int_{-\infty}^{\infty} \mathcal{E}_{m}^{*}\left(\mathbf{r}_{\perp}\right) \mathcal{E}_{n}^{*}\left(\mathbf{r}_{\perp}\right) \mathrm{d} \mathbf{r}_{\perp}=\delta_{m, n} .
$$


The propagation constants of the array modes, $\beta$, are determined by the solution of the eigenvalue equation

$$
(\mathbf{C}+i \beta \mathbf{I}) \mathbf{E}=0
$$

where $\mathbf{I}$ is the $N \times N$ unit matrix, and $\omega$ represents the frequency of interest.

Implicit in Eq. (4) is the boundary condition $c_{0}=c_{N+1}$ $=0$ (which corresponds to no field propagating in the two end waveguides). Furthermore, we assume the $s$ th mode of the waveguide array takes the form $\mathcal{E}^{(s)}(\mathbf{r})$ $=\Sigma_{n=1}^{N} a_{n}^{(s)} \mathcal{E}_{n}(x, z) \exp \left(-i \beta^{(s)} y\right)$. The associated eigenmodes and propagation constants are given by

$$
\begin{aligned}
& \beta^{(s)}=\beta_{0}+M_{l}+2 \kappa_{l} \cos \left(\frac{s \pi}{N+1}\right), \\
& a_{n}^{(s)}=\sin \left(\frac{n s \pi}{N+1}\right),
\end{aligned}
$$

where $s=1, \ldots, N$ and $n=1, \ldots, N$ are integers and $n$ enumerates positions of the waveguides.

Thus far, the coupled-mode theory we have used is in the spatial domain where we have solved for the $N$ propagation constants of the waveguide array supermodes [13], $\beta^{(s)}$, at a fixed (given) frequency embodied by $\beta_{0}$ $=\omega n_{\text {eff }}(\omega) / c$. If we introduce reflection at the end of each waveguide in an array of resonators, the feedback along the $y$ direction discretizes $\beta^{(s)}$ such that the modes of the resonator arrays satisfy $\beta^{(s)} L+\phi_{0}=m \pi$, where $L$ is the length of the array length in $y, \phi_{0}$ is the phase shift incurred at each reflector, and $m$ is an integer. Hence, to find the dispersion relationship of a resonator array, we use Eq. (8a) to determine the frequencies (or values of $\beta_{0}$ ) for which $\beta^{(s)} L+\phi_{0}=m \pi$. For the calculations that follow, we will set $\phi_{0}$ to zero to simplify the expressions, but this phase shift can be easily accounted for in the results by subtracting $\phi_{0}$ wherever $m \pi$ appears.

Figure 2 clarifies the effects of imposing an additional set of boundary conditions in $y$ to the coupled-mode treatment of an array of waveguides. For the calculations, we have assumed eight coupled resonators with a coupling constant of $8 \times 10^{-4} \mu \mathrm{m}^{-1}$. Without the feedback in the $y$ direction, we compute $N=8$ values of $\beta^{(s)}$ at each frequency, thus arriving at the linear dispersion relations (the sloped lines) shown on the left half of the figure. The condition $\beta^{(s)} L=m \pi(L=500 \mu \mathrm{m}, m=2097)$, denoted by the vertical line in the plot, "selects" the resonance frequencies of the resonator array, which are marked by “ $\times$ "s. These resonance frequencies in turn correspond to particular values of $s \pi /(N+1)$ shown on the right side of Fig. 2. Through this process, the waveguide dispersion relations from the time-independent coupled-mode theory are converted to the dispersion relation of the coupled resonators, and the relation between eigenmode splitting in the time-independent coupled-mode theory and the modes of a CROW is highlighted.

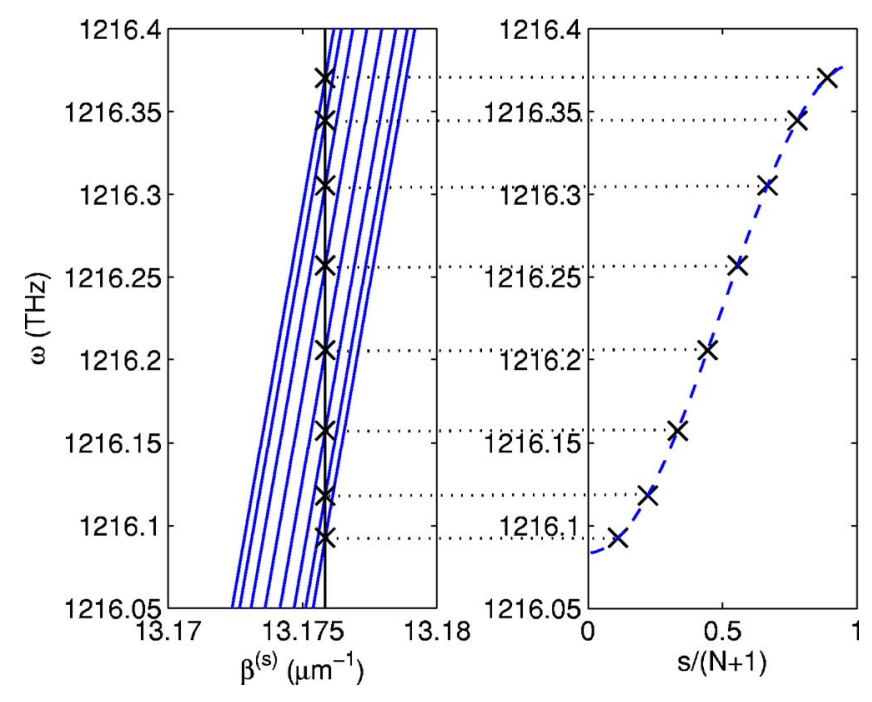

Fig. 2. (Color online) Schematic illustrating the role of the additional resonance or boundary condition in $y$. On the left, the resonance condition $\beta^{(s)} L=m \pi$ selects the resonance frequencies from the dispersion relations of the waveguide array. These frequencies correspond to particular values of $s \pi /(N+1)$ on the right.

Assuming that $\Omega$ is the resonance frequency of an uncoupled resonator, such that $\beta_{0}(\Omega) L=\Omega n_{\mathrm{eff}}(\Omega) L / c=m \pi$, setting $\beta^{(s)} L=m \pi$ gives

$$
\omega^{(s)}=\Omega \frac{n_{\mathrm{eff}}(\Omega)}{n_{\mathrm{eff}}\left(\omega^{(s)}\right)}\left[1-\frac{M_{l} L}{m \pi}-2 \frac{\kappa_{l} L}{m \pi} \cos \left(\frac{s \pi}{N+1}\right)\right] .
$$

For $N$ coupled cavities, there are $N$ discrete resonant frequencies.

As $N \rightarrow \infty$, the array modes described by Eq. (9) form a continuum and the array eigenmodes of the structure can be treated as the Bloch modes of the system. A Bloch mode is a periodic function in which the field in the $(n$ +1 )th period differs from the $n$th period by a phase factor of $K \Lambda$, where $K$ is the (continuous) Bloch wavenumber and $\Lambda$ is the period. Because the fields described by Eq. (8b) are standing waves along $z$, they can be decomposed into superposition of counterpropagating traveling waves along $\hat{z}$. Therefore, by comparison with Eq. (8b), we can replace

$$
\lim _{N \rightarrow \infty} s \pi /(N+1) \rightarrow K \Lambda
$$

This leads to the dispersion of a CROW based on an array of coupled Fabry-Perot resonators

$$
\omega(K)=\Omega\left[1-\frac{M}{m \pi}-2 \frac{\kappa}{m \pi} \cos (K \Lambda)\right],
$$

where we have assumed $n_{\text {eff }}(\omega)=n_{\text {eff }}(\Omega)=n$ is a constant, and $\kappa_{l} L=\kappa$ and $M_{l} L=M$ are dimensionless coupling coefficients. The frequency dependence of $\kappa$ and $M$ are given by Eq. (5); however, since the bandwidth of a CROW is not expected to be large $(\omega / \Omega \ll 1)$, the coupling coefficients can be assumed to be constant.

The dispersion relation described by Eq. (11) is of the 
same form as the CROW dispersion from the tightbinding approximation and the dispersion calculated using transfer matrices for ring resonators [14]. The key difference between Fabry-Perot and ring resonators is that only two $K$ vectors correspond to a particular eigenfrequency for the Fabry-Perot resonators while there are four $K$ vectors for the rings. Physically, this is because a ring resonator supports two degenerate modes on resonance (i.e., even and odd, or clockwise and anticlockwise), while a Fabry-Perot resonator supports one mode on resonance.

The slowing factor, given by the ratio of the speed of light to the maximum group velocity in the CROW, is

$$
S=\frac{c}{v_{\left.g\right|_{\max }}}=\frac{n L}{2 \kappa \Lambda}=\frac{n}{2 \kappa_{l} \Lambda} .
$$

Unlike coupled grating defects or ring resonators, the pe$\operatorname{riod} \Lambda$ of the CROW is decoupled from $L$. Since for weakly coupled single-mode waveguides $\kappa_{l} \approx 10^{-4}-10^{-3} \mu \mathrm{m}^{-1}$ and $\Lambda$ can be $\sim 5 \mu \mathrm{m}$ even for modest index contrast $(\Delta n / n$ $\approx 10^{-3}-10^{-2}$ ), large slowing factors of the order of a few hundred to a thousand are possible.

\section{TRANSFER MATRIX ANALYSIS}

While the modes and the dispersion relation of a waveguide array CROW can be determined from the coupledmode theory of a waveguide array, the transmission spectrum does not immediately follow from the calculations. One approach to calculate the spectrum is to expand the input excitation field in terms of the eigenmodes of the CROW and propagate the modes individually. A second, more convenient approach, which we shall describe in this section, is to use a transfer matrix formalism. This method can account for an arbitrary input excitation at the array end facet, applies to asymmetric structures, and can also be used for arrays with side-coupled inputoutput waveguides.

To use the transfer matrix formalism, we describe the propagation of light through the structure with a $2 N$ $\times 2 N$ matrix. The matrix acts on a column vector describing both the forward and backward propagating fields at each waveguide as shown in Figs. 1(c) and 1(d). We denote the fields at each interface by

$$
\begin{aligned}
& \mathbf{U}(y)=\left[E_{1}^{(+)}(y) E_{2}^{(+)}(y) \ldots E_{N}^{(+)}(y)\right]^{T}, \\
& \mathbf{D}(y)=\left[E_{1}^{(-)}(y) E_{2}^{(-)}(y) \ldots E_{N}^{(-)}(y)\right]^{T},
\end{aligned}
$$

where $E_{n}^{(+)}(y)$ and $E_{n}^{(-)}(y)$ are the forward and backward propagating fields, respectively, in each element at a particular value of $y$. Thus, the fields at $y=L$ are related to those at $y=0$ by

$$
\left[\begin{array}{l}
\mathbf{U}(L) \\
\mathbf{D}(L)
\end{array}\right]=\mathbf{S}^{(2)} \mathrm{Q} \mathbf{S}^{(1)}\left[\begin{array}{c}
\mathbf{U}(0) \\
\mathbf{D}(0)
\end{array}\right] .
$$

Here $\mathbf{S}^{(1,2)}$ are matrices describing the reflectors at $y=l_{1}$ and $y=l_{2}$, which can arise from Fresnel or Bragg reflection. Q is the transfer matrix that describes the coupling and propagation in the array region.

To simplify the numerics and make the system more tractable, we assume that light is not coupled from one element to the next in the reflector sections (from $y=0$ to $y=l_{1}$ and from $y=l_{2}$ to $\left.y=L\right)$. This assumption is valid for reflection from cleaved facets and for well-confined waveguide modes in short gratings. For the intercavity coupling (from $y=l_{1}$ to $y=l_{2}$ ), we are primarily interested in the weak coupling regime, where only nearest-neighbor coupling is significant. The inclusion of the more general effect (e.g., nearest-neighbor coupling in the grating sections) will lead to quantitative, but not qualitative, changes in our results.

The form of $\mathbf{S}^{(q)}$ (where $q=1,2$ ) is given by

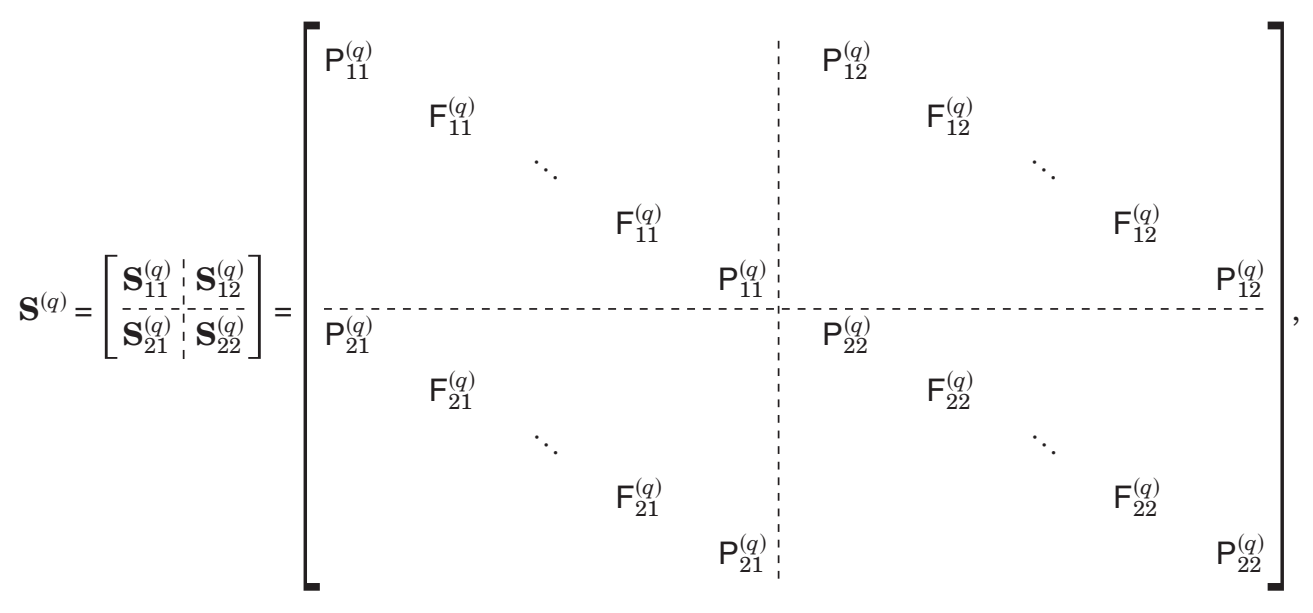


where $\mathbf{S}_{i j}^{(q)}$ are $N \times N$ diagonal submatrices, $\mathrm{P}_{i j}^{(q)}$ are $2 \times 2$ transfer matrices for the input and output waveguides or resonators, and $F_{i j}^{(q)}$ are derived from the $2 \times 2$ transfer matrices for the reflectors. In other words, for the middle waveguides (where $n \neq 1, N$ ) we have

$$
\begin{aligned}
& {\left[\begin{array}{l}
E_{n}^{(+)}\left(l_{1}\right) \\
E_{n}^{(-)}\left(l_{1}\right)
\end{array}\right]=\left[\begin{array}{ll}
\mathrm{F}_{11}^{(1)} & \mathrm{F}_{12}^{(1)} \\
\mathrm{F}_{21}^{(1)} & \mathrm{F}_{22}^{(1)}
\end{array}\right]\left[\begin{array}{l}
E_{n}^{(+)}(0) \\
E_{n}^{(-)}(0)
\end{array}\right],} \\
& {\left[\begin{array}{l}
E_{n}^{(+)}(L) \\
E_{n}^{(-)}(L)
\end{array}\right]=\left[\begin{array}{ll}
\mathrm{F}_{11}^{(2)} & \mathrm{F}_{12}^{(2)} \\
\mathrm{F}_{21}^{(2)} & \mathrm{F}_{22}^{(2)}
\end{array}\right]\left[\begin{array}{l}
E_{n}^{(+)}\left(l_{2}\right) \\
E_{n}^{(-)}\left(l_{2}\right)
\end{array}\right] .}
\end{aligned}
$$

For an array of $N-2$ resonators with sidecoupled waveguides for input and output coupling, $P_{12}^{(q)}=P_{21}^{(q)}=0$ and $P_{11}^{(q)}=\left(P_{22}^{(q)}\right)^{-1}$ describes the accumulation of phase. This leads to $\mathrm{P}_{11}^{(1)}=\exp \left(-i \beta_{0} l_{1}\right)$ and $\mathrm{P}_{11}^{(2)}=\exp \left[-i \beta_{0}\left(L-l_{2}\right)\right]$. Otherwise, for input coupling at the end facet of an array of $N$ resonators, $\mathrm{P}_{i j}^{(q)}=\mathrm{F}_{i j}^{(q)}$. The elements $\mathrm{F}_{i j}^{(q)}$ can be readily calculated for an arbitrary type of mirror (e.g., Bragg reflectors, cleaved facets).

In the waveguide-coupler section of the structure in Figs. 1(c) and 1(d), one can use the coupled-mode theory described in Section 2:

$$
\frac{d}{\mathrm{~d} y}\left[\begin{array}{l}
\mathbf{U}(y) \\
\mathbf{D}(y)
\end{array}\right]=\left[\begin{array}{ll}
\mathbf{C} & \varnothing \\
\varnothing & \mathbf{C}^{\dagger}
\end{array}\right]\left[\begin{array}{l}
\mathbf{U}(y) \\
\mathbf{D}(y)
\end{array}\right] .
$$

Therefore,

$$
\begin{aligned}
{\left[\begin{array}{l}
\mathbf{U}\left(l_{2}\right) \\
\mathbf{D}\left(l_{2}\right)
\end{array}\right] } & =\left[\begin{array}{ll}
\mathbf{Q} & \varnothing \\
\varnothing & \mathbf{Q}^{\dagger}
\end{array}\right]\left[\begin{array}{l}
\mathbf{U}\left(l_{1}\right) \\
\mathbf{D}\left(l_{1}\right)
\end{array}\right] \equiv \mathrm{Q}\left[\begin{array}{l}
\mathbf{U}\left(l_{1}\right) \\
\mathbf{D}\left(l_{1}\right)
\end{array}\right], \\
\mathbf{Q} & =\exp (\mathbf{C} L) .
\end{aligned}
$$

Combining Eqs. (15) and (19), the transfer matrix for the overall system is given by

$$
\begin{aligned}
{\left[\begin{array}{l}
\mathbf{U}(L) \\
\mathbf{D}(L)
\end{array}\right] } & =\left[\begin{array}{ll}
\mathbf{S}_{11}^{(2)} & \mathbf{S}_{12}^{(2)} \\
\mathbf{S}_{21}^{(2)} & \mathbf{S}_{22}^{(2)}
\end{array}\right]\left[\begin{array}{ll}
\mathbf{Q} & \varnothing \\
\varnothing & \mathbf{Q}^{\dagger}
\end{array}\right]\left[\begin{array}{ll}
\mathbf{S}_{11}^{(1)} & \mathbf{S}_{12}^{(1)} \\
\mathbf{S}_{21}^{(1)} & \mathbf{S}_{22}^{(1)}
\end{array}\right]\left[\begin{array}{l}
\mathbf{U}(0) \\
\mathbf{D}(0)
\end{array}\right] \\
& \equiv\left[\begin{array}{ll}
\mathbf{G}_{11} & \mathbf{G}_{12} \\
\mathbf{G}_{21} & \mathbf{G}_{22}
\end{array}\right]\left[\begin{array}{l}
\mathbf{U}(0) \\
\mathbf{D}(0)
\end{array}\right],
\end{aligned}
$$

where $\mathbf{G} \equiv \mathbf{S}^{(2)} \mathrm{Q} \mathbf{S}^{(1)}$.

Rearranging terms in Eq. (20) and assuming $\mathbf{D}(L)=0$ (no field is incident from the right), we have

$$
\begin{aligned}
& \mathbf{U}(L)=\left(\mathbf{G}_{11}-\mathbf{G}_{12} \mathbf{G}_{22}^{-1} \mathbf{G}_{21}\right) \mathbf{U}(0), \\
& \mathbf{D}(0)=\left(-\mathbf{G}_{22}^{-1} \mathbf{G}_{21}\right) \mathbf{U}(0),
\end{aligned}
$$

which relates the input and output fields of our structure.

The transfer matrices can account for an arbitrary input field at $y=0$ and can be used to calculate the reflection and transmission coefficients of any resonator. However, in most cases, we are primarily interested in exciting the first element and the transmission and reflection coefficients in the first and last elements only. In this case, the boundary conditions are $\mathbf{U}^{T}(0)=\left[\begin{array}{llllll}1 & 0 & 0 & 0 & \ldots & 0\end{array}\right]$ and $\mathbf{D}^{T}(L)$ $=\left[\begin{array}{lllll}0 & 0 & 0 & 0 & \ldots\end{array}\right]$. Using Eq. (21) and the boundary conditions, the transmission and reflection coefficients are

$$
\begin{aligned}
& R_{1}=\frac{D_{1}(0)}{U_{1}(0)}, \quad R_{N}=\frac{D_{N}(0)}{U_{1}(0)}, \\
& T_{1}=\frac{U_{1}(L)}{U_{1}(0)}, \quad T_{N}=\frac{U_{N}(L)}{U_{1}(0)},
\end{aligned}
$$

with $U_{n}(y)$ representing the $n$th element of $\mathbf{U}(y)$, and $R_{1}$ and $T_{1}$ are the reflection and transmission coefficients at the input-through port as marked in Fig. 1, while $R_{2}$ and $T_{2}$ are the coefficients at the drop-output port.

The transmission and reflection spectra for a CROW with five resonators with side-coupled input and output waveguides are shown in Fig. 3. The reflectors in the calculations consist of Bragg gratings with alternating layers of thicknesses $d_{H}=119 \mathrm{~nm}$ and $d_{L}=123 \mathrm{~nm}$, with effective indices $n_{H}=3.25$ and $n_{L}=3.15$, respectively. The gratings are $24 \mu \mathrm{m}$ or 100 periods long. The waveguide sections have an effective index of 3.25 and are $50 \mu \mathrm{m}$ long. The coupling constant is $\kappa_{l}=4 \times 10^{-3} \mu \mathrm{m}^{-1}$. These parameters can be accomplished by $1.25 \mu \mathrm{m}$ wide waveguides with an effective index of 3.25 spaced about $\sim 900 \mathrm{~nm}$ apart surrounded by a cladding of index 3.15 . The resultant length of the coupled resonators in the direction of periodicity is about $\sim 10 \mu \mathrm{m}$.

By design, the standing-wave cavities supports a resonance mode at a free-space wavelength of $1.551 \mu \mathrm{m}$. It is
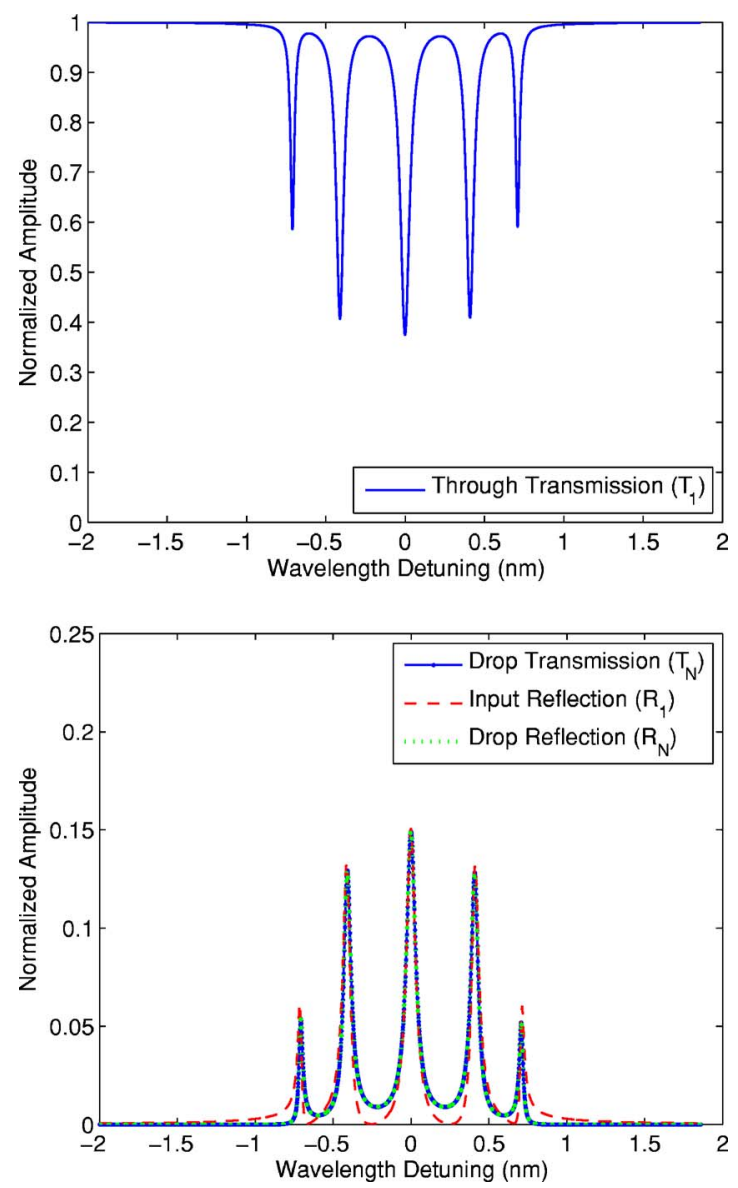

Fig. 3. (Color online) (a) Transmission spectrum at the through port and (b) the transmission and reflection spectra at the input and drop ports for the side-coupled array. 
apparent from Fig. 3 that the transmission properties of our structures resemble that of microring CROWs [14]. In close vicinity of the resonance frequency $\Omega$, the transmission across cavities is increased. In contrast to CROWs consisting of traveling wave cavities (e.g., ring-disk resonators), the maximum transmission in the present situation is $25 \%$ rather than unity as in the case of ring resonators. This is attributed to the fact that the standingwave cavity has no degenerate modes at $\Omega$, and the fields in the cavity can decay into the two waveguides in both the forward and backward directions [15]. This poses a limitation on the practicality of a passive system, but will not be a main concern for systems with optical gain. In passive systems, the maximum transmission shown in Fig. 3(b) can be improved by increasing the reflectivity of the Bragg gratings through increasing the number of periods and/or the index contrast. The ripples in the spectrum can be reduced by apodizing the coupling constants [16-18].

\section{OPTICAL GAIN}

The most straightforward implementation of the FabryPerot CROWs is an array of waveguides with cleaved facets providing the feedback for the resonators. Since the Fresnel reflection coefficient is only $\sim 30 \%$, a large optical gain is necessary to compensate for the losses. Gain introduces an imaginary component to the coupling constants, $\kappa_{l}$ and $M_{l}$, and can be used to tune the CROW dispersion if the gain-loss modulation is strong [19].

Optical amplification (and loss) can be built into the coupled-mode theory by writing the dielectric constant as a complex function:

$$
\epsilon(\mathbf{r})=\bar{\epsilon}(\mathbf{r})+\sum_{n=1}^{N}\left[\Delta \epsilon(\mathbf{r}-n \Lambda)+i \frac{2 \epsilon_{\mathrm{eff}}}{\beta_{0}} \Delta \gamma(\mathbf{r}-n \Lambda)\right],
$$

where $\Delta \gamma$ denotes the gain coefficient in the waveguides, and $\epsilon_{\text {eff }}$ normalizes $\Delta \gamma / \beta_{0}$ to $\epsilon(\mathbf{r})$ and is the effective dielectric constant of the waveguides. The gain is a periodic function in $z$ in practice because the gain in the waveguide core and cladding areas will not be identical.

We assume the uncoupled modes of the individual waveguides are $\mathcal{E}_{n}(x, z) \exp \left(-i \beta_{0} y\right)$. Using the normalization condition [Eq. (6)], the coupling constants in the presence of gain are

$$
\begin{aligned}
\widetilde{\kappa}_{l} & =\kappa_{l}+i \frac{\omega \epsilon_{0} \epsilon_{\mathrm{eff}}}{2 \beta_{0}} \sum_{j=1}^{N} \int_{-\infty}^{\infty} \mathcal{E}_{n}^{*}\left(\mathbf{r}_{\perp}\right) \Delta \gamma(\mathbf{r}-j \Lambda) \mathcal{E}_{n+1}\left(\mathbf{r}_{\perp}\right) \mathrm{d} \mathbf{r}_{\perp} \\
& \equiv \kappa_{l}+i \kappa_{l}^{\prime}, \\
\tilde{M}_{l} & =M_{l}+i \frac{\omega \epsilon_{0} \epsilon_{\mathrm{eff}}}{2 \beta_{0}} \sum_{j=1}^{N} \int_{-\infty}^{\infty} \mathcal{E}_{n}^{*}\left(\mathbf{r}_{\perp}\right) \Delta \gamma(\mathbf{r}-j \Lambda) \mathcal{E}_{n}\left(\mathbf{r}_{\perp}\right) \mathrm{d} \mathbf{r}_{\perp} \\
& \equiv M_{l}+i M_{l}^{\prime},
\end{aligned}
$$

where $\kappa_{l}$ and $M_{l}$ are the coupling constants in the passive structure and are given in Eq. (5).

The coupling coefficients are now complex. In typical semiconductor materials, $\Delta \gamma$ is of the order of $10^{-3}-10^{-2} \mu \mathrm{m}^{-1}$, while $\beta_{0}$ is of the order of $10 \mu \mathrm{m}^{-1}$. On the other hand, even in low-index contrast systems, $\Delta \epsilon$ is of the order of $10^{-1}(\Delta n \sim 0.01)$. Therefore, in most cases, $\kappa_{l}^{\prime} / \kappa_{l}, M_{l}^{\prime} / M_{l} \ll 1$.

If the Bloch vectors are complex (i.e., to account for net gain-loss in the direction of propagation), $K=K_{R}+i K_{I}$, then the dispersion relation for the CROW is

$$
\begin{aligned}
\omega(K)= & \Omega\left[1-\frac{M}{m \pi}-2 \frac{\kappa}{m \pi} \cos \left(K_{R} \Lambda\right) \cosh \left(K_{I} \Lambda\right)\right. \\
& \left.-2 \frac{\kappa^{\prime}}{m \pi} \sin \left(K_{R} \Lambda\right) \sinh \left(K_{I} \Lambda\right)\right] .
\end{aligned}
$$

$K_{I}$ can be determined from the net gain of the supermodes of the waveguide array:

$$
\begin{aligned}
\gamma(K)= & M_{l}^{\prime}+2 \kappa_{l}^{\prime} \cos \left(K_{R} \Lambda\right) \cosh \left(K_{I} \Lambda\right) \\
& -2 \kappa_{l} \sin \left(K_{R} \Lambda\right) \sinh \left(K_{I} \Lambda\right) .
\end{aligned}
$$

Approximating the gain of the supermodes is roughly equal to the gain of the individual waveguides, $\gamma(K)$ $\approx M_{l}^{\prime}, K_{I} \Lambda$ is given by

$$
\operatorname{coth}\left(K_{I} \Lambda\right)=\frac{\kappa_{l}}{\kappa_{l}^{\prime}} \tan \left(K_{R} \Lambda\right),
$$

where $K_{I} \Lambda=0$ when $K_{R} \Lambda=0, \pi / 2, \pi$.
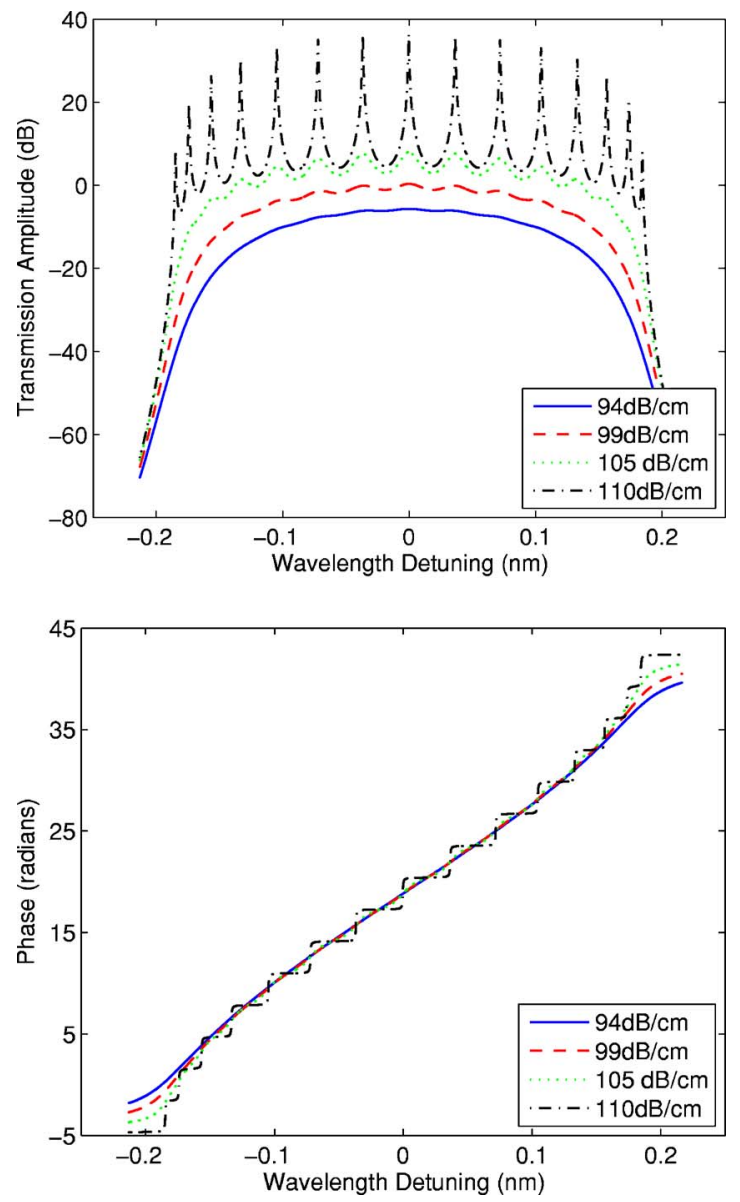

Fig. 4. (Color online) (a) Transmission and (b) phase responses of a resonator array for various gain values. The input is end coupled into the first element of the array. 
Near the band center, which is the frequency range of interest as the CROW group-velocity dispersion is minimum, $K_{I} \Lambda$ is of the order of $10^{-3}$ and is negligibly small. Hence, the dispersion relation does not change significantly with gain. However, in real systems, the refractive index is expected to change with the gain through thermal and carrier injection effects. If these effects induce a large gain-loss modulation in the CROW, then the dispersion relation can be significantly modified with the optical gain [19].

In the approximation that the coupling constant remains constant with gain, the gain can be modeled in the transfer matrices by the inclusion of a complex propagation constant $\beta_{0}+i \gamma_{0}$. Figure 4 shows the transmission and phase responses of the drop port (or the last resonator) for various values of gain calculated using the transfer matrices by adding an imaginary component to the propagation constant for an array of 15 resonators. The input is end coupled into first element of the array as in Fig. 1(d) and the output is taken from the last resonator. The length of the waveguides is $500 \mu \mathrm{m}$, and the coupling constant is $8 \times 10^{-4} \mu \mathrm{m}^{-1}$. The gain values correspond to $85 \%, 90 \%, 95 \%$, and $99.8 \%$ of the mirror losses $1 /(2 L) \ln \left(r^{2}\right)$, where $r^{2}=0.28$ is about the reflectivity of cleaved facets. The coupling strength can be achieved with $3 \mu \mathrm{m}$ wide waveguides separated by about $\sim 1 \mu \mathrm{m}$ with an index contrast of $\Delta n / n \sim 0.05$, resulting in slowing factors of about 600 .

\section{CONCLUSION}

We have presented a means of slowing light with lowindex contrast CROWs using coupled waveguide and laser resonator arrays. Low-index contrast systems have the advantage of having smaller sidewall scattering losses for a given roughness and typically requiring simpler fabrication processes (e.g., larger feature sizes, shallower etch depth). We have analyzed evanescently coupled arrays and show that they can achieve slowing factors of several hundreds times with bandwidths of tens of gigahertz. Optical amplification, naturally present in laser arrays, overcomes the severe limitation of high optical attenuation characteristic of most passive slow light structures. Combining evanescent coupling in the propagation direction with Bragg or Fresnel reflection in the orthogonal direction provides an approach for engineering more complex periodic structures to slow light.

\section{ACKNOWLEDGMENTS}

J. Poon and P. Chak are grateful for the support from the Natural Sciences and Engineering Research Council of Canada. J. Choi thanks the U.S. Department of Defense and the American Society for Engineering Education for their support. Financial support from the National Sci- ence Foundation (award 0438038) and the Defense Advanced Research Projects Agency (Slow Light Project) are gratefully acknowledged.

\section{REFERENCES}

1. A. Yariv, Y. Xu, R. K. Lee, and A. Scherer, "Coupledresonator optical waveguide: a proposal and analysis," Opt. Lett. 24, 711-713 (1999).

2. G. Lenz, B. J. Eggleton, C. K. Madsen, and R. E. Slusher, "Optical delay lines based on optical filters," IEEE J. Quantum Electron. 37, 525-532 (2001).

3. S. Lan, S. Nishikawa, H. Ishikawa, and O. Wada, "Engineering photonic crystal impurity bands for waveguides, all-optical switches and optical delay lines," IEICE Trans. Electron., E85C, 181-189 (2002).

4. H. Altug and J. Vuckovic, "Experimental demonstration of the slow group velocity of light in two-dimensional coupled photonic crystal microcavity arrays," Appl. Phys. Lett. 86, 111102 (2005).

5. F. N. Xia, L. Sekaric, and Y. Vlasov, "Ultracompact optical buffers on a silicon chip," Nat. Photonics 1, 65-71 (2007).

6. M. Bayindir, S. Tanriseven, and E. Ozbay, "Propagation of light through localized coupled-cavity modes in onedimensional photonic band-gap structures," Appl. Phys. A 72, 117-119 (2001).

7. S. Olivier, C. Smith, M. Rattier, H. Benisty, C. Weisbuch, T. Krauss, R. Houdre, and U. Osterle, "Miniband transmission in a photonic crystal waveguide coupledresonator optical waveguide," Opt. Lett. 26, 1019-1051 (2001).

8. B. E. Little, S. T. Chu, P. P. Absil, J. V. Hryniewicz, F. G. Johnson, F. Seiferth, D. Gill, V. Van, O. King, and M. Trakalo, "Very high-order microring resonator filters for WDM applications," IEEE Photon. Technol. Lett. 16, 2263-2265 (2004)

9. J. K. S. Poon, L. Zhu, G. A. DeRose, and A. Yariv, "Transmission and group delay in microring coupledresonator optical waveguides," Opt. Lett. 31, 456-458 (2006).

10. A. Scherer, O. Painter, B. D'Urso, R. Lee, and A. Yariv, "InGaAsP photonic band gap crystal membrane microresonators," J. Vac. Sci. Technol. B 16, 3906-3910 (1998).

11. U. Peschel, O. Egorov, and F. Lederer, "Discrete cavity solitons," Opt. Lett. 29, 1909-1911 (2004).

12. D. Botez and D. R. Scifres, Diode Laser Arrays (Cambridge U. Press, 1994).

13. A. Yariv, Optical Electronics in Modern Communications, 5th ed. (Oxford U. Press, 1997).

14. J. K. S. Poon, J. Scheuer, S. Mookherjea, G. T. Paloczi, Y. Huang, and A. Yariv, "Matrix analysis of microring coupled-resonator optical waveguides," Opt. Express 12, 90-103 (2004).

15. S. Fan, P. R. Villeneuve, J. D. Joannopoulos, and H. A. Haus, "Channel drop filters in photonic crystals," Opt. Express 3, 4-11 (1998).

16. C. K. Madsen and J. H. Zhao, Optical Filter Design and Analysis: a Signal Processing Approach (Wiley, 1999).

17. M. Sumetsky and B. J. Eggleton, "Modeling and optimization of complex photonic resonant cavity circuits," Opt. Express 11, 381-391 (2003).

18. P. Chak and J. E. Sipe, "Minimizing finite-size effects in artificial resonance tunneling structures," Opt. Lett. 13, 2568-2570 (2006)

19. S. Mookherjea, "Using gain to tune the dispersion relation of coupled-resonator optical waveguides," IEEE Photon. Technol. Lett. 18, 715-717 (2006). 